\title{
Programa Nutricional para a Melhoria da Saúde do Trabalhador por meio da Adição de Alimento Funcional
}

\author{
Ludmila C. Borges, Ana Paula C. Silveira, Christiane A. Starling \& \\ Karolline F. Siqueira
}

Com o objetivo de desenvolver receitas à base de soja e introduzi-las na dieta dos trabalhadores da empresa Genix Indústria Farmacêutica Ltda., foi aprovado no Edital SENAI SESI de Inovação no ano de 2009 o projeto denominado "Desenvolvimento de programa nutricional para a melhoria da saúde do trabalhador, por meio da adição de alimento funcional à dieta - equilibra". O desenvolvimento do projeto se deu por meio da parceria firmada entre a empresa, o SENAI e o SESI do Estado de Goiás. O objetivo principal teve como foco a promoção de soluções inovadoras para a segurança e saúde na indústria.

Palavras-chave: proteína; soja, receituários; formulações à base de soja.

With the aim of developing soy-based recipes and introduce them in the diet of the company's workers Genix Pharma Ltda., Was approved in the Notice SENAI Innovation SESI in 2009 the project called "nutritional program development for the improvement of worker health by the addition of functional food diet - balance ". The project development was through the partnership between the company, SENAI and SESI the State of Goiás. The main objective was focused on promoting innovative solutions for safety and health in the industry.

Keywords: protein; soy; prescriptions; soy formulations. 


\section{Introdução}

Lançado em 2004, o Edital SENAI SESI de Inovação já aprovou 686 projetos em parceria com 589 empresas industriais brasileiras e startups de base tecnológica, com investimentos de mais de R\$ 380 milhões em projetos inovadores, até o ano de 2015. O objetivo do Edital é financiar o desenvolvimento de novos produtos, processos e serviços inovadores da indústria nacional. O desenvolvimento é realizado em conjunto com o SENAI ou SESI, dependendo do escopo do projeto. Os projetos selecionados por meio do Edital devem promover o aumento da competitividade e da produtividade industrial em um cenário global, por meio da inovação tecnológica e da promoção de soluções inovadoras para a segurança e saúde na indústria.

Denominado como "Desenvolvimento de programa nutricional para a melhoria da saúde do trabalhador por meio da adição de alimento funcional à dieta - equilibra", este foi aprovado no Edital SENAI SESI de Inovação no ano de 2009 e desenvolvido em conjunto com o SENAI e SESI do estado de Goiás, tendo em vista que o objetivo principal teve como foco a promoção de soluções inovadoras para a segurança e saúde na indústria.

$\mathrm{O}$ objetivo do projeto foi desenvolver receitas à base de soja e introduzi-las na dieta dos trabalhadores, por meio de um programa que englobasse ações educativas, $o$ enriquecimento da alimentação com alimento funcional e o monitoramento por meio de análises laboratoriais, com vista na melhoria da qualidade de vida do trabalhador da Indústria.

A resolução No 18 de 30 de abril de 1999, da ANVISA ${ }^{1}$, define alegação de propriedade funcional como "aquela relativa ao papel metabólico ou fisiológico que o nutriente ou não nutriente tem no crescimento, desenvolvimento, manutenção e outras funções normais do organismo humano". A mesma resolução define que "o alimento ou ingrediente que alegar propriedades funcionais ou de saúde pode, além de funções nutricionais básicas, quando se tratar de nutriente, produzir efeitos metabólicos e ou fisiológicos e ou efeitos benéficos à saúde, devendo ser seguro para consumo sem supervisão médica".

Segundo CHIARELLO $(2002)^{2}$, atualmente, a soja é a cultura que mais impacta o PIB (Produto Interno Bruto) agrícola brasileiro. Dos R $\$ 55$ bilhões alcançados pela agricultura em 2001, R \$ 14 bilhões foram representados por produtos em grãos, farelo e óleo. Durante os últimos 25 anos, em virtude dos investimentos públicos feitos em P\&D (Pesquisa e Desenvolvimento), principalmente, por meio da Empresa Brasileira de Pesquisa Agropecuária - EMBRAPA, foi possível a adaptação e introdução da soja em praticamente todas as regiões do país.

Mais de $90 \%$ da produção dos insumos utilizados na produção animal e na indústria de óleos e gorduras são representados pela soja. Já com relação à leguminosa, em escala reduzida, esta é utilizada na obtenção de produtos tradicionais da cultura oriental, como leite de soja, tofu, natto, misso e shoyu. Na busca por produtos de maior valor agregado, a soja surge como matéria-prima de outras indústrias de alimentos, empregando-a como ingrediente funcional e/ou nutricional para a produção de farinhas, proteínas texturizadas, concentradas e isoladas (CHIARELLO, 2002) ${ }^{2}$.

Desde a Antiguidade, é reconhecida a importância da alimentação na manutenção da saúde. Recentes descobertas no campo nutricional, relacionando hábitos alimentares com a incidência de doenças, estão mudando os paradigmas dos consumidores e das indústrias de alimentos. É visível a preocupação crescente com a alimentação e sua relação com saúde e longevidade, principalmente, nos países desenvolvidos, criando um grande mercado para alimentos que possam beneficiar a saúde. Partindo deste princípio, um assunto bastante discutido trata-se da definição de alimentos funcionais que, de forma genérica, são caracterizados como os alimentos ou ingredientes alimentares que podem acarretar benefícios à saúde, além de suas propriedades nutricionais, quando consumidos como parte de uma dieta saudável. Neste contexto, a partir de estudos recentes, uma série de potenciais benefícios para a saúde podem estar relacionados a componentes da soja, proporcionando com que a soja e seus derivados façam parte dos ingredientes de maior sucesso na fabricação de alimentos funcionais. Entre os potenciais benefícios, destacam os efeitos preventivos em doenças cardiovasculares, osteoporose e câncer, além do alívio dos sintomas da menopausa (CHIARELLO, 2002) ${ }^{2}$.

As proteínas são componentes da dieta, necessárias à sobrevivência de homens e animais, tendo como função primordial suprir o organismo de aminoácidos essenciais (OETTERER, et al. , 2006) ${ }^{4}$. A soja é uma planta 
leguminosa, rica em proteínas, de alto valor biológico, e que representa uma alternativa importante na alimentação humana, podendo estar presente na mesa da maioria das famílias. A soja e seus derivados são alguns dos ingredientes de maior sucesso na fabricação de alimentos com alegação de propriedades funcionais. Estudos recentes apontam uma série de potenciais benéficos para a saúde, que podem estar relacionados a componentes da soja. Os altos níveis de colesterol sanguíneo e do LDLcolesterol (low density lipoprotein, cholesterol) estão associados às doenças cardiovasculares, tais como o infarto do miocárdio e a arteriosclerose, e também tem sido demonstrada a relação entre DAC (Doença Arterial Coronariana) e concentrações séricas reduzidas de HDL-colesterol (high density lipoprotein, cholesterol). A participação dos triglicerídeos na aterogênese ainda permanece em discussão, porém se justifica a dosagem sérica de rotina desse lípide, devido à sua frequente vinculação à doença cardiovascular aterosclerótica e a outros distúrbios metabólicos. Pesquisas da American Heart Association (Associação Americana do Coração) têm demonstrado que a ingestão de proteínas de soja reduzem as taxas de LDL-colesterol (CHIARELLO, $2002)^{2}$.

O consumo diário de no mínimo $25 \mathrm{~g}$ de proteína de soja pode ajudar a reduzir o colesterol. Seu consumo deve estar associado a uma alimentação equilibrada e hábitos de vida saudáveis. (BRASIL, 2008) .No $^{1}$ Brasil, apesar dos números superlativos em relação ao volume de produção da leguminosa, a falta de hábito da população em consumir alimentos contendo soja e o desconhecimento de potenciais benefícios relacionados à sua inclusão na dieta resultam em um mercado ainda muito incipiente. (CHIARELLO, 2002) ${ }^{2}$. Diante desta realidade, nota-se a importância proporcionada pelo desenvolvimento do projeto.

O trabalho foi realizado como parte do Projeto de Inovação Tecnológica do Edital de Inovação SESI/ SENAI 2009, e em parceria com a Empresa Genix Indústria Farmacêutica Ltda. Instalada em Anápolis, Goiás, o principal polo farmacêutico do Brasil, a Genix Indústria Farmacêutica Ltda. consolida a sua participação no mercado desde 2001 como um dos maiores fabricantes de cápsulas de gelatina duras vazias do mundo. É considerada por especialistas do segmento farmacêutico como a fábrica modelo na América Latina, devido à utilização dos melhores padrões de tecnologia internacional. Seus equipamentos são importados do Canadá e possuem tecnologia avançada e grande capacidade produtiva, além de uma equipe profissional que garante a qualidade dos produtos. A empresa buscou o desenvolvimento deste projeto, vislumbrando o aumento da competitividade e da produtividade industrial em um cenário global, e a segurança e saúde dos trabalhadores.

A Empresa Genix Indústria Farmacêutica Ltda. busca se aperfeiçoar nos quesitos inovação e produtividade. NAPOLITANO et. al. $(2013)^{3}$ define inovação tecnológica como a introdução de um novo produto ou processo no mercado, ou de uma versão melhorada dos já existentes. Segundo os autores a empresa Genix Indústria Farmacêutica Ltda. foi parceira de um projeto que teve como objetivo apresentar um produto inovador no mercado nacional de filmes plásticos, criado a partir da transformação de resíduos derivados da cápsula para medicamento farmacêutico.

O objetivo do projeto foi desenvolver receitas a base de soja e introduzi-las na dieta dos trabalhadores, por meio de um programa que englobasse ações educativas, $o$ enriquecimento da alimentação com alimento funcional e o monitoramento por meio de análises laboratoriais, com vistas na melhoria da qualidade de vida do trabalhador da indústria.

\section{Métodos}

O Projeto teve andamento juntamente com o Programa Cozinha Brasil (SESI), que proporcionou a participação de nutricionistas responsáveis pelo programa. O cozinha Brasil teve o objetivo de oferecer cursos de curta e média duração, gratuitos à população, para o ensino da prática de uma alimentação nutritiva e saudável, de baixo custo e que respeita as diferenças regionais e das estações. Além de receitas fáceis e saborosas, os aprendizes também foram capacitados a: escolher os alimentos por seu valor nutritivo, preço e apresentação; aproveitar integralmente os alimentos, reduzindo o desperdício; preparar refeições observando a limpeza e preservando o sabor e os nutrientes dos alimentos; consumir alimentos em quantidade adequada 
e sob condições de higiene; e promover a melhoria da renda familiar.

Durante a sensibilização dos funcionários, foram realizadas várias palestras com temas direcionados à melhoria da saúde do trabalhador, além da elaboração de folders e banners para orientações de uma alimentação saudável. $\mathrm{O}$ desenvolvimento do receituário à base de soja foi realizado pela equipe SENAI e pela equipe do Cozinha Brasil do SESI. Os resultados obtidos foram satisfatórios.

Foi realizada uma seleção das preparações já servidas, selecionadas as mais aceitas pelos funcionários da empresa, e reformuladas incluindo derivados de soja como ingrediente principal. Além destas, foram desenvolvidas novas formulações à base de soja.

Os ingredientes funcionais, utilizados na elaboração das formulações, foram: proteína texturizada clara e escura (PTS), extrato de soja, kinako (farinha torrada) e soja em grãos. Esses foram submetidos à análise de proteína para verificação do teor de proteína em cada insumo. Estas análises foram realizadas pelo Laboratório de Controle de Qualidade de Alimentos da Faculdade de Farmácia da Universidade Federal de Goiás. Os receituários e formulações foram desenvolvidos nas unidades do SENAI e SESI Vila Canaã e por meio do Programa Cozinha Brasil. Para cada formulação foi elaborada uma ficha técnica de preparação, constando todos os ingredientes, quantidades e o modo de preparo.

$\mathrm{Na}$ elaboração dos cardápios, foram incluídas receitas doces e salgadas e distribuídas durante o período de trabalho dos funcionários da empresa, com maior ênfase no almoço, ocasião em que o funcionário pode ingerir a quantidade correta de proteína de forma mais eficaz.

Todas as preparações foram elaboradas com o objetivo da ingestão mínima diária de 25 gramas de proteína de soja por pessoa, conforme o FDA (Food and Drug Administration). Assim, a ingestão de soja auxilia na redução do risco de doenças cardiovasculares, pela diminuição dos níveis de colesterol. Dessa forma, esperase uma melhoria na saúde do trabalhador, contribuindo para a minimização do índice de abstinência no trabalho.

A análise sensorial de cada preparação foi realizada por uma equipe não treinada, com um teste de aceitação, pela equipe do SESI e SENAI. Foi observado que a maioria das formulações não apresentava o gosto residual da soja, permitindo que a aceitabilidade de cada produto fosse garantida.

\section{ETAPAS DO PROJETO}

Para o desenvolvimento do projeto, foram previstas a execução das seguintes etapas: Elaboração do material promocional (Folders de divulgação do projeto; Brindes; Banners); Lançamento da campanha na empresa (Degustação de produtos à base de soja; Palestra de apresentação); Primeira realização de exames laboratoriais com todos os funcionários da empresa e primeira aplicação de anamnese com todos os funcionários da empresa; Seleção do grupo de trabalho; Assinatura do termo de consentimento livre e esclarecido; Sensibilização dos funcionários participantes do projeto por meio de palestras; Desenvolvimento das preparações à base de soja e análise sensorial; Elaboração dos cardápios; Início dos cardápios na empresa e segunda realização de exames laboratoriais com o grupo. Segunda avaliação nutricional com o grupo selecionado por meio da aplicação do processo de anamnese; Curso do Programa Cozinha Brasil com os funcionário e familiares na empresa Genix Indústria Farmacêutica Ltda.; Palestras sobre alimentação e hábitos de vida saudáveis; Terceira e quarta realização de exames laboratoriais com o grupo selecionado e avaliação nutricional com o grupo selecionado; Elaboração da caixa de receitas saudáveis; Tabulação dos resultados, classificação dos ganhadores, premiação pela empresa para os participantes do projeto, confecção dos cheques simbólicos e encerramento do projeto na empresa com a entrega da caixa de receitas.

\section{DESENVOLVIMENTO DO PROJETO}

As primeiras atividades executadas foram a elaboração de material promocional com o objetivo de distribuir aos funcionários da empresa Genix Indústria Farmacêutica Ltda. para a divulgação do projeto. A Figura 1 apresenta os brindes produzidos para a divulgação. Além desta ação, o lançamento da campanha na empresa se deu, também, por meio da degustação de produtos à base de soja, conforme ilustra a Figura 1. Além disso, houve uma palestra com a finalidade de esclarecer o contexto do programa no qual a empresa foi inserida. 

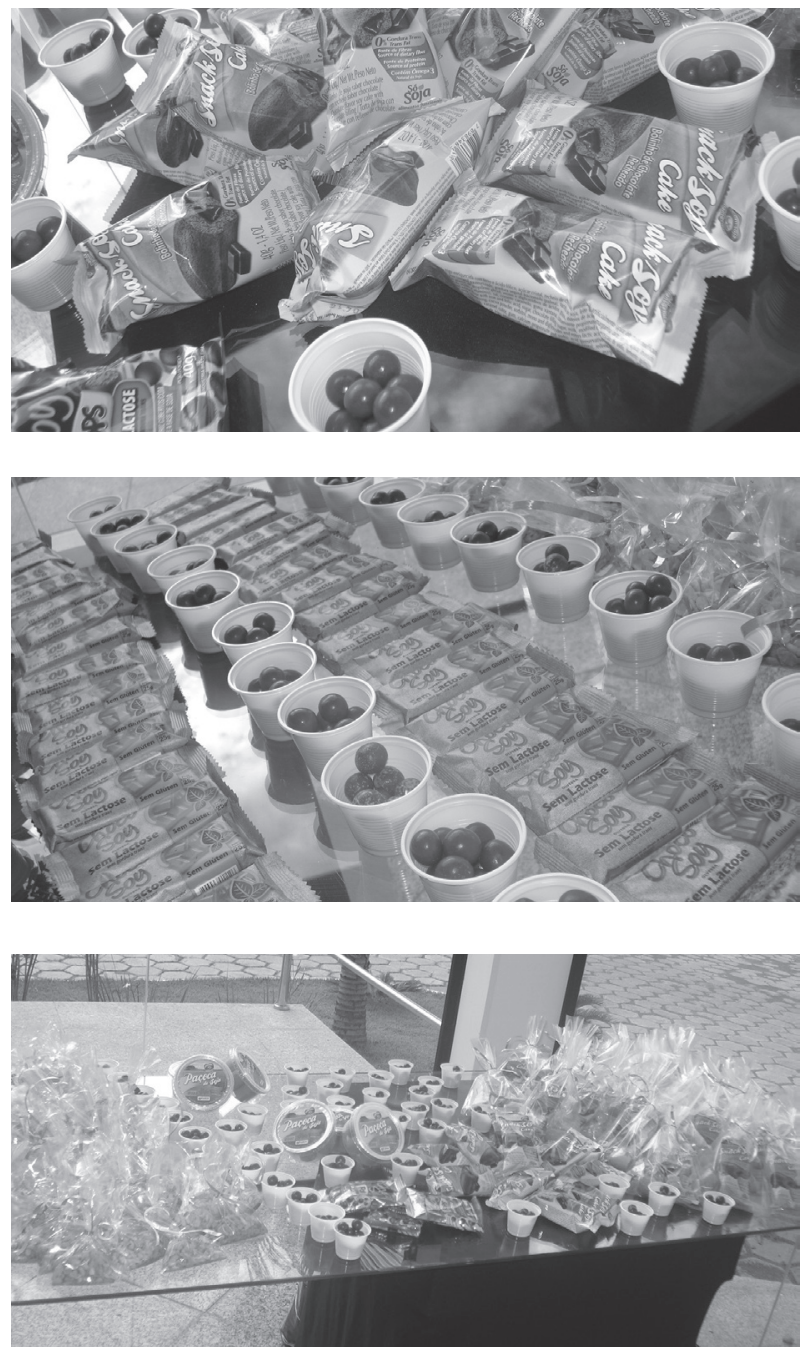

Figura 1. Lançamento do programa na empresa: degustação de produtos a base de soja

Em um segundo momento, realizaram-se exames laboratoriais em todos os funcionários da empresa e foi aplicado, também, o processo denominado "anamnese". Segundo SANTOS, VEIGA, ANDRADE (2010) $)^{5}$, a anamnese é definida como a primeira fase de um processo, na qual a coleta dos dados permite ao profissional de saúde identificar problemas, determinar diagnósticos, planejar e implementar a sua assistência. Para alguns autores, devem ser coletados quatro tipos de dados nessa primeira fase que são: subjetivos, objetivos, históricos e atuais, que podem ser obtidos por meio de entrevista, observação, exame físico, resultados de provas diagnósticas, revisão de prontuário e pela colaboração de outros profissionais. Além dos exames laboratoriais, foi realizada a aferição de medidas dos funcionários, que constitui um dos dados que compõem o processo de anamnese.

Diante dos dados coletados, foi possível realizar a seleção do grupo de funcionários que participou do programa. $\mathrm{O}$ critério de seleção baseou-se nos seguintes requisitos pré-estabelecidos no projeto: colesterol acima de $180 \mathrm{mg} / \mathrm{dl}$ (segundo Diretrizes da Sociedade Brasileira de Cardiologia); IMC maior ou igual a $25 \mathrm{~kg} / \mathrm{m}^{2}$ (limite inferior da faixa limítrofe, associado a fatores de risco para desenvolvimento de doenças cardiovasculares); e, em alguns casos, com triglicérides elevado.

Após a realização dos exames, foi constatado que 61 funcionários se adequaram aos requisitos préestabelecidos no projeto, os quais se submeteram à assinatura de um termo de consentimento livre e esclarecido para a inserção destes no programa. Em seguida, o próximo passo foi a sensibilização do grupo selecionado por meio de três palestras voltadas aos seguintes temas: atualidades na alimentação; doenças causadas por má alimentação e dietas da moda.

Em paralelo à sensibilização do grupo selecionado, foram desenvolvidas preparações à base de soja, seguida de análise sensorial. Diante de tais resultados, a equipe responsável pelo desenvolvimento do projeto elaborou cardápios diferenciados para a inserção das preparações à base de soja nas refeições dos funcionários da empresa. A inserção das preparações se deu no café da manhã, almoço, sobremesa e lanche. Após tal ação, foram realizadas avaliações periódicas (exames laboratoriais e aplicação de anamnese) a cada dois meses, em todo o grupo selecionado, totalizando o quantitativo de quatro avaliações.

Como forma de beneficiar os funcionários mais comprometidos com o programa, vislumbrando o empenho de cada participante, foi prevista uma premiação pela empresa aos cinco participantes melhor classificados ,quanto à redução do nível de colesterol total durante o desenvolvimento do programa. Por fim, como entrega final do projeto, foi desenvolvida uma caixa de receitas à base de soja para os funcionários darem continuidade ao consumo de soja. 


\section{Resultados}

Os resultados do projeto foram avaliados em função das etapas previstas e do objetivo geral estabelecido. Para a inserção de formulações à base de soja nas refeições dos funcionários da empresa, foram desenvolvidos cardápios diferenciados, apresentados na Figura 2.
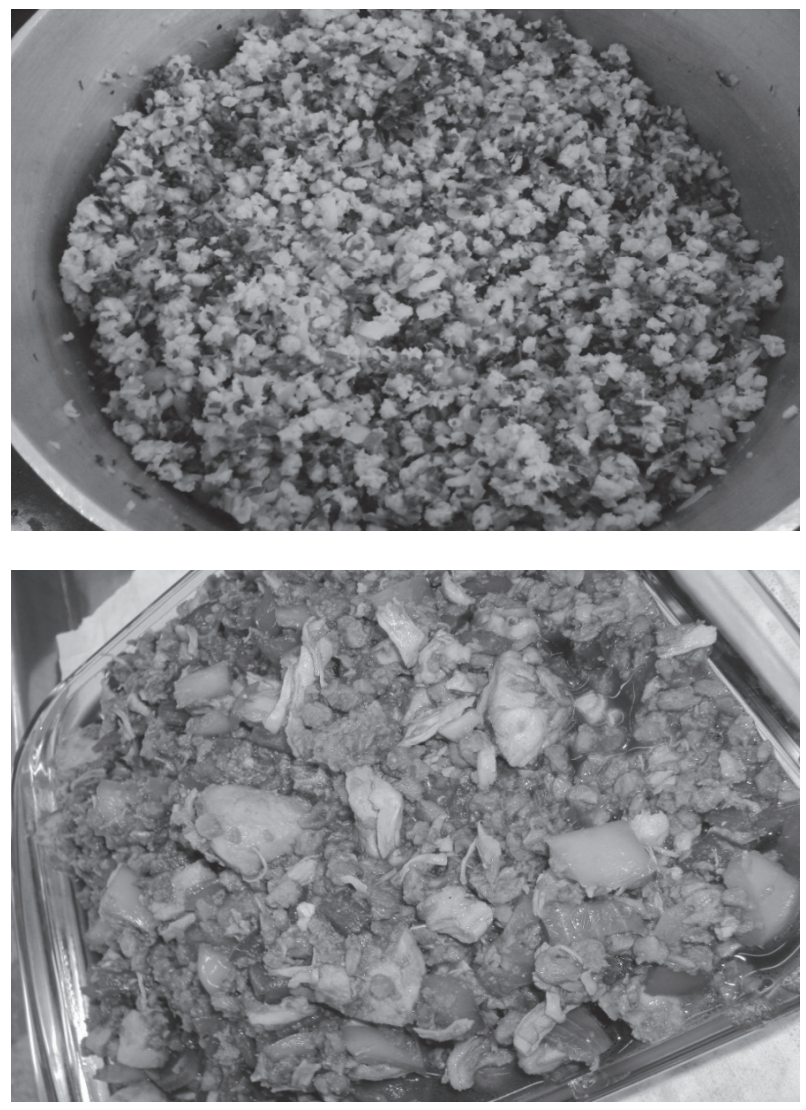

Figura 2. Frango xadrez com proteína texturizada e recheio de lasanha à base de proteína com brócolis

Sendo um dos objetivos do projeto o desempenho dos funcionários quanto à redução do nível de colesterol total, os resultados dos exames do primeiro e do quinto funcionário melhor classificados. Foi possível observar que $36,40 \%$ dos participantes reduziram os níveis de colesterol total e 49,09\% reduziram o IMC (Índice de Massa Corporal). O Figura 3 apresenta o resultado dos cinco funcionários melhor classificados quanto à redução do nível de colesterol total.

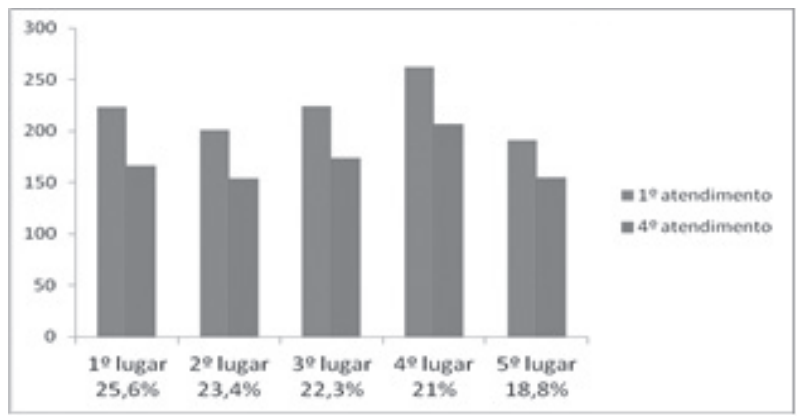

Figura 3. Resultado dos cinco funcionários melhor classificados

Alguns dados importantes observados após o término do programa referem-se à diminuição do absenteísmo dos funcionários assim como à melhora da disposição dos colaboradores que participaram do programa. Segundo dados levantados, o número de funcionários da empresa, em 2009, era de 282, tendo um aumento para 305 funcionários no ano de 2010. No mesmo período, foi observado que, em 2009, a empresa teve 147 casos de absenteísmo, enquanto que em 2010 este número foi reduzido para 113 casos. Fazendo uma relação entre os dados coletados, observa-se o índice de absenteísmo de $52,13 \%$ no ano de 2009 , o qual este mesmo índice foi reduzido para 37,05\% no ano de 2010. Como consequência de tais resultados, tem-se o aumento da produtividade da indústria. Como entrega final do projeto, foi elaborado e distribuído um receituário com todas as preparações à base de soja, desenvolvidas no projeto, conforme apresentado na Figura 4.

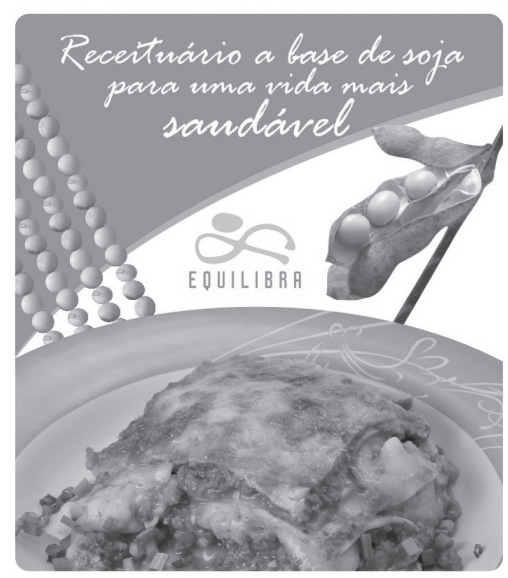

Figura 4. Entrega final do projeto: caixa de receitas a base de soja 


\section{Conclusão}

Tendo em vista que faz parte dos objetivos do SESI e do SENAI desenvolver ações que viabilizem a aproximação Instituição/Indústria, e que os projetos selecionados por meio do Edital SENAI SESI de Inovação devem promover o aumento da competitividade e da produtividade industrial em um cenário global, por meio da inovação tecnológica e da promoção de soluções inovadoras para a segurança e saúde na indústria, pode-se dizer que o projeto foi um sucesso.

A indústria ganhou ao ter colaboradores mais envolvidos, motivados e comprometidos. Do outro lado, os colaboradores ganharam por se sentirem inseridos dentro do processo organizacional do qual fazem parte e pela qualidade de vida obtida com o desenvolvimento do projeto. Em relação ao SESI e ao SENAI, o ganho diz respeito ao exercício de sua missão e visão e maior visibilidade e credibilidade junto aos seus clientes, acometidas a partir de um produto viável. Como resultado, foi obtida a redução do nível de colesterol dos funcionários participantes da pesquisa, sendo significativa em relação ao número de participantes.

O desenvolvimento do projeto impactou positivamente nas ações de responsabilidade social da empresa Genix Indústria Farmacêutica Ltda., o qual contribuiu fortemente para que a indústria obtivesse boa avaliação no PSQT - Prêmio SESI Qualidade no Trabalho, que homenageia as indústrias brasileiras que investem na valorização e na qualidade de vida dos trabalhadores. Pioneiro no setor, o Prêmio estimula o exercício da cidadania nas relações de trabalho e incentiva as empresas a incorporarem a responsabilidade social em suas estratégias, compartilhando boas práticas.

Por fim, tendo em vista o objetivo do projeto, podese concluir que este foi um sucesso, pois as ações educativas e a introdução de receitas à base de soja na dieta dos colaboradores, tendo como consequência o enriquecimento da alimentação com alimento funcional, permitiram a melhoria da qualidade de vida do trabalhador da Indústria.

\section{Referências}

1. Agência Nacional De Vigilância Sanitária. $N^{\circ} 18$. Diretrizes básicas para análise e comprovação de propriedades funcionais e ou de saúde alegadas em rotulagem de alimentos. 1999.

2. Chiarello, M. D. Soja e os Alimentos Funcionais - Encontro Franco Brasileiro de Biociência e Biotecnologia - Alimentos Funcionais e Nutracêuticos, Brasília, 2002, p.7. (Embrapa. Documentos, 85).

3. Napolitano, H.; Afonso, F.; Carvalho, V.; Teixeira, I. Bioprodutos a partir de Resíduos da Produção de Cápsulas Derivadas de Gelatina Bovina. Revista Processos Químicos. Jul/Dez de 2013.

4. Oetterer, M.; Regitano-D'arce, M. A. B.; Spoto, M. H. F. Fundamentos de Ciência e Tecnologia de Alimentos. Barueri, SP: Manole, 2006. 632 p.

5. Santos, N.; Veiga, P.; Andrade, R. Importância da anamnese e do exame físico para o cuidado do enfermeiro. Revista Brasileira de Enfermagem - REBEn. Centro Universitário Jorge Amado, Salvador, BA, 2010.

\section{Ludmila C. Borges, Ana Paula C. Silveira*, Christiane A. Starling \& Karolline F. Siqueira}

Instituto SENAI de Tecnologia em Alimentos e Bebidas,-- Rua Professor Lázaro Costa n 348, Vila Canaã, CEP: 74415-420, Goiânia, Goiás, Brasil.

*E-mail: anasilveira.senai@sistemafieg.org.br 